\section{High mobility group \\ A-interacting proteins in cancer: focus on chromobox protein homolog 7 , homeodomain interacting protein kinase 2 and PATZ}

\author{
Monica Fedele, \\ Giovanna Maria Pierantoni, \\ Pierlorenzo Pallante, Alfredo Fusco
}

Istituto di Endocrinologia e Oncologia Sperimentale (IEOS) CNR, Dipartimento di Biologia e Patologia Cellulare e Molecolare, Università di Napoli Federico II, Naples, Italy

\section{Abstract}

The High Mobility Group A (HMGA) proteins, a family of DNA architectural factors, by interacting with different proteins play crucial roles in neoplastic transformation of a wide range of tissues. Therefore, the search for HMGA-interacting partners was carried out by several laboratories in order to investigate the mechanisms underlying HMGA-dependent tumorigenesis. Three of the several HMGAbinding proteins are discussed in this review. These are the Chromobox family protein (chromobox protein homolog 7, CBX7), the homeodomain interacting protein kinase 2 (HIPK2) and the POZ/domain and Kruppel zinc finger family member, PATZ. All of them play a critical role in tumorigenesis, and may also be independent markers of cancer. Their activities are linked to cell cycle, apoptosis and senescence. In this review, we discuss the properties of each protein, including their effect on HMGAl functions, and propose a model accounting for how their activities might be coordinated.

\section{Introduction}

The high mobility group A (HMGA) family is comprised of three low molecular weight proteins: HMGA1a, HMGA1b, and HMGA2 (formerly HMGI, HMGY, and HMGI-C, respectively). They are encoded by two distinct genes, HMGA1 and HMGA2, which are located on human chromosomal band 6p21 and chromosomal region $12 q 13-15$, respectively. These proteins bind the minor groove of AT-rich DNA sequences through three short basic repeats, the so-called AT-hooks and orchestrate the assembly of nucleoprotein complexes through a high-grade network of protein-DNA and pro- tein-protein interactions, playing key roles in chromatin architecture, gene transcription and replication. These functions have important rebounds in a wide spectrum of biological processes, ranging from embryonic development, cell differentiation and transformation, cell cycle progression, apoptosis, senescence, DNA repair, up to different aspects of cell physiopathology. ${ }^{1,2}$ Both HMGA genes are widely and abundantly expressed during embryogenesis, conversely the expression of HMGA2 has not been detected in any of the several adult mouse and human tissues tested, is very low in CD34 positive hematopoietic stem cells, in mouse preadipocytic proliferating cells and in meiotic and post-meiotic cells (secondary spermatocytes and spermatids). ${ }^{3-6}$ Just recently, HMGA2 expression has emerged to have a central role in self-renewal and maintenance of the undifferentiated state (stemness) in embryonic as well as adult, normal and cancer stem cells. ${ }^{7,8}$ As far as HMGA1 is concerned, its expression in adult tissues is much lower in comparison with that observed in embryonic tissues. $^{3}$

Overexpression of $H M G A 1$ and HMGA2 represents a general feature of experimental and human malignancies. However, no mutations or rearrangements have been detected in human carcinomas apart from a few cases of hematological neoplasias where alterations of HMGA2 have been reported. In malignant tumors of a wide range of tissues, including thyroid, colon, pancreatic, breast, lung and gastric cancer, a clear association between high HMGA1/A2 expression and a poor prognosis has been also observed, suggesting detection of HMGA proteins as diagnostic molecular marker and predictor of poor post-operative survival. ${ }^{9}$

The HMGA genes have also a critical role in the generation of human benign tumors of mesenchymal origin in which the HMGA2 gene is rearranged. The effects of such rearrangements are dysregulation of the HMGA2 gene, its expression, truncation, or formation of fusion genes encoding chimeric transcripts containing the three AT-hooks of HMGA2 and ectopic sequences from other genes. In some cases only few amino acids are fused to the HMGA2 DNA binding domains, suggesting that the loss of the HMGA2 sequences rather than the acquisition of new sequences is important for HMGA2 oncogenic activity. ${ }^{10,11}$ Recent studies evidence that the loss of the 3 ' untranslated region (3' UTR) contributes to the HMGA2 oncogenic activity. Indeed, the HMGA2 3' UTR contains target sequences for different microRNAs (miRs), such as let-7, miR-98, and miR-196. Since miRs can down-regulate gene expression at the post-transcription level by binding to seed sequences located in the 3'UTR region of the target genes and causing
Correspondence: Monica Fedele, Istituto di Endocrinologia e Oncologia Sperimentale IEOS CNR, via S. Pansini, 5 - 80131, Napoli, Italy.

Tel. +39.081.746.3054 - Fax: +39.081 .746 .3749 .

E-mail:mfedele@unina.it

Key words: High Mobility Group, cancer, CBX7, HIPK2, PATZ1.

Acknowledgements: the authors are supported by grants from the Associazione Italiana per la Ricerca sul Cancro (IG 5728 - IG 11477 - MFAG 11702) and Programmi di Ricerca Scientifica di Rilevante Interesse Nazionale (PRIN 2009).

Contributions: MF, wrote the abstract, the introduction, the section on PATZ and coordinated the full manuscript; GMP, wrote the abstract, the introduction and the section on HIPK2; PP, wrote the section on $\mathrm{CBX7}$; $\mathrm{AF}$ wrote the introduction, the section on $\mathrm{CBX7}$ and the conclusions.

Received for publication: 25 November 2011.

Revision received: 6 February 2012.

Accepted for publication: 13 February 2012.

This work is licensed under a Creative Commons Attribution NonCommercial 3.0 License (CC BYNC 3.0).

(C) Copyright M. Fedele et al., 2012

Licensee PAGEPress, Italy

Journal of Nucleic Acids Investigation 2012; 3:e1 doi:10.4081/jnai.2012.e1

either cleavage of the mRNA or inhibition of protein synthesis, the truncation of the HMGA2 gene, with the consequent loss of its 3'UTR, would result in increased HMGA2 protein levels that would have oncogenic activity. ${ }^{12-14}$ More recently, similar miR-mediated mechanisms have been also investigated for the $H M G A 1$ gene. ${ }^{15,16}$

Therefore, the up-regulation of HMGA protein expression appears a common event in benign as in malignant tumors, but the different mechanisms of involvement of HMGA in malignant or benign tumors (overexpression versus rearrangement) are not established yet. It is now clearly established that the overexpression of HMGA in different types of cancer is not casually associated to cell transformation, but plays a causal role in tumor development. Indeed, in vitro studies have shown that blocking the synthesis of HMGA proteins can either prevent neoplastic transformation or lead neoplastic cells to death. ${ }^{17}$ Furthermore, overexpression of HMGA proteins transforms cells in vitro and determines the development of several forms of neoplasias in vivo, including lipomas, NK-T/NK and T cell lymphomas, uterine leiomyomas, fibroadenomas of the breast, salivary gland adenomas and mixed growth hormone/prolactin cell pituitary adenomas. ${ }^{18,19}$ 
The mechanisms by which HMGA proteins are implicated in neoplastic transformation are multiple and include enhancement of cell proliferation, escape from apoptosis, impairment of DNA repair, induction of inflammatory response and modulation of EpithelialMesenchymal Transition (EMT). ${ }^{9}$ Since the HMGAs are mainly involved in transcriptional regulation and their role depends on the interaction with other proteins, one of the main approaches aimed at identifying the mechanisms by which these proteins could be involved in cancer has been the search for HMGA-interactors. Several complementary approaches were used to give a set of HMGA interactors for some of which relevant functional data have been obtained: co-immunoprecipitation, affinity chromatography, twodimensional gel electrophoresis, mass spectrometry, yeast two-hybrid and antibody arrays. ${ }^{20-24}$ A list of some HMGA-binding proteins is shown in Table $1 .^{23-37}$ This list only includes proteins of which a cancer-related function is known. Interactions such as these, as well as post-translational modifications discussed elsewhere in this issue, frequently dictate how HMGA proteins behave. For example, some proteins function as transcriptional coactivators of HMGA, such as p300/CBP, while others appear to dictate the nature of HMGAdownstream response. Indeed, a common feature of HMGA-binding proteins seems the ability to determine the transforming potential of the HMGA proteins. It is now clear that HMGA proteins are able to establish several connections within the cell, depending on the cellular context. This network suggests an involvement of these proteins in many processes, such as transcription, DNA replication, chromatin remodeling and mRNA processing. ${ }^{2,20-22,25}$

It is beyond the scope of any review to discuss adequately all of the many different HMGA-binding proteins, and do them justice. Therefore, we have concentrated our attention on a few interesting and novel proteins, and which are a focus of our laboratories.

\section{Cromobox protein homolog 7}

Chromobox protein homolog 7 (CBX7) belongs to the chromobox protein family and is a member of the polycomb repressive complex 1 (PRC1) that is involved in the modulation of the developmental regulatory genes. ${ }^{38-40}$ In this context it has been found that $\mathrm{CBX} 7$ could trigger epigenetic change in the CDKN2a-locus (trimethylation of H3K9) through the recruitment and activation of SUV39H2, with the fol- lowing binding of PRC1 within the silenced regions of euchromatin, leading to a fully epigenetic inactivation of p16 by modifications of histone. ${ }^{41}$ Mouse $c b x 7$ is able to associate with the inactive $\mathrm{X}$ chromosome and with the facultative heterochromatin thereby implicating that CBX7 protein could act as a transcriptional repressor if it is localized in silenced chromatin. ${ }^{42,43}$

It has been recently reported that $\mathrm{CBX} 7$ is drastically downregulated in thyroid carcinomas and its expression progressively decreases with malignant grade and neoplastic stage. Indeed, CBX7 protein levels decreased in an increasing percentage of cases going from benign adenomas to papillary, follicular and anaplastic thyroid carcinomas. ${ }^{44}$ However, the correlation of the loss of CBX7 with a highly malignant phenotype and a consequent poor prognosis appears to be a general event in oncology. In fact, the loss of CBX7 expression has been recently shown to be associated with increasing malignancy grade in colon, ${ }^{45}$ pancreatic, ${ }^{46}$ bladder ${ }^{47}$ breast, ${ }^{48}$ gastric, ${ }^{49}$ and lung carcinoma, ${ }^{30}$ whereas the retention of CBX7 expression correlates with a longer survival. ${ }^{45}$ Consistent with these findings, it has been recently demonstrated that $\mathrm{CBX7}$ is able to positively regulate the expression of the $E$-cadherin gene. ${ }^{50}$ This gene plays a critical role in maintaining normal epithelial cell morphology, and loss of its expression represents a feature of the EMT. ${ }^{51,52}$ CBX7 is able to preserve the expression of $E$-cadherin by interacting with histone deacetylase 2 (HDAC2) and inhibiting its activity on the E-cadherin gene promoter. ${ }^{50}$ As confirmation of this mechanism, a direct correlation between the levels of E-cadherin and CBX7 either in thyroid carcinomas than in those of the pancreas has been found. ${ }^{46,50}$ Moreover, CBX7 is also able to negatively regulate the expression of other important genes involved in EMT. Preliminary data produced in our laboratory show that $\mathrm{CBX} 7$ is able to repress the expression of $S 100 A 4$ and osteopontin, two genes essential for the acquisition of the transformed phenotype. ${ }^{53,54}$ Thus, the loss of the expression of CBX7 can represent a central node in directing the EMT, as its reduced expression may directly lead to the deregulated expression of a series of important genes (Figure 1A).

Even though all these results propose CBX7 as a tumor suppressor gene, and suggest a critical role of CBX7 downregulation in the acquisition of the neoplastic phenotype, other studies report that $\mathrm{CBX} 7$ could act as an oncogene. In fact, it has been demonstrated that it cooperates with c-Myc to generate highly aggressive B-cell lymphomas and can start T-cell lymphomagenesis. $^{39}$ Moreover, it has been

Table 1. Cancer-related High Mobility Group A-interacting proteins.

\begin{tabular}{|c|c|c|}
\hline $\begin{array}{l}\text { Protein } \\
\text { name }\end{array}$ & Function & Reference \\
\hline AP-1 & transcriptional regulation & 26 \\
\hline ATF-2 & transcriptional regulation & 27 \\
\hline ATM & DNA-repair & 28,29 \\
\hline CBX7 & chromatin remodeling & 30 \\
\hline Fra-2 & transcriptional regulation & 31 \\
\hline FUS & $\begin{array}{c}\text { DNA-repair } \\
\text { transcriptional regulation } \\
\text { cytoplasm export } \\
\text { RNA splicing }\end{array}$ & 21 \\
\hline HIPK2 & $\begin{array}{l}\text { protein-kinase } \\
\text { cell cycle } \\
\text { apoptosis }\end{array}$ & 23 \\
\hline Nek2 & $\begin{array}{l}\text { protein-kinase } \\
\text { cell cycle }\end{array}$ & 32 \\
\hline NF-kB & transcriptional regulation & 27 \\
\hline PATZ & transcriptional regulation & 33 \\
\hline $\operatorname{PPAR} \gamma$ & transcription & 34 \\
\hline $\mathrm{pRb}$ & cell cycle & 35,36 \\
\hline p53 & $\begin{array}{l}\text { transcriptional regulation } \\
\text { cell cycle } \\
\text { apoptosis }\end{array}$ & 24,37 \\
\hline $\mathrm{p} 63$ & transcriptional regulation & 37 \\
\hline RAR $\alpha$ & transcriptional regulation & 34 \\
\hline RNF4 & $\begin{array}{c}\text { transcriptional regulation } \\
\text { protein ubiquitination }\end{array}$ & 33 \\
\hline
\end{tabular}


observed that CBX7 can prolong the lifetime of several normal human cells and immortalize mouse fibroblasts by a mechanism involving a downregulation of Ink4a/Arf. ${ }^{55,56} \mathrm{On}$ the other hand the silencing of CBX7 expression by RNAi methodology causes an inhibition of the normal cell growth by the induction of Ink4a/Arf. ${ }^{56}$

The recent generation of knockout mice for the $C b x 7$ gene seems to validate the tumor suppressor role of the $C B X 7$ gene, since these mice develop liver and lung adenomas and carcinomas. $^{30}$ This study reports that the CBX7 protein interacts with the HMGAl protein on the promoter of the gene coding for cyclin $\mathrm{E}$ (CCNE1) participating in the DNA-bound multimeric complex containing also HDAC2. In particular, it has been seen that CBX7 is able to recruit HDAC2 on the $C C N E 1$ promoter, competing with HMGA1, that, conversely, displaces the CBX7/HDAC2 complex from this promoter. Therefore, CBX7 binds the CCNE1 promoter and inhibits $C C N E 1$ transcription counteracting the enhancement of the CCNE1 transcription by HMGAlb. ${ }^{30}$ By the mean of this mechanism involving the displacement of HMGA1, CBX7 triggers histone acetylation at CCNE1 promoter, and the increased Cyclin E expression likely accounts for the neoplastic phenotype of the cbx7-knockout mice since a very similar neoplastic pathology has been described in transgenic mice overexpressing cyclin E. ${ }^{57}$ Interestingly, HMGA1 is able to negatively regulate the expression of $\mathrm{CBX7}$, as it has been seen in breast carcinomas. ${ }^{48}$ In addition, HMGA1 is also able of inducing the expression of miR-181b, which is in turn contrasted by CBX7. CBX7 is a molecular target of miR-181b and, therefore, it is clear that in this way a feedback loop is generated (Figure 1B). ${ }^{47}$

\section{Homeodomain-interacting protein kinase-2}

Ten years ago, in order to isolate proteins interacting with the HMGAl proteins, a yeast two-hybrid screening was performed using the HMGAlb protein as bait. This analysis led to the isolation of Homeodomain-interacting protein kinase-2 (HIPK2). ${ }^{23}$ HIPK2 is a member of a recently identified serine-threonine kinase family, as belonging to a family of transcriptional co-repressors during the development. ${ }^{58}$ HIPK2 protein consists of 1189 amino acids, and contains a protein kinase catalytic domain, an interaction domain for homeoproteins, a co-repressor domain, a PEST sequence, a speckle-retention signal and an autoinhibitory domain in the $\mathrm{COOH}$-terminal. ${ }^{58}$ The interaction between HIPK2 and HMGA1 occurs through the PEST domain of
HIPK2 and the second AT-hook domain of HMGA1 (Figure 2A) ${ }^{23}$ Since kinase activity is frequently required for HIPK2-mediated regulation of gene transcription and the phosphorylation of HMGA1 proteins was associated with cellular transformation and proliferation, ${ }^{59}$ the capability of HIPK2 to phosphorylate HMGA1 proteins has been further investigated. Three sites of HMGAla protein Ser-35, Thr-52, and Thr-77 were phosphorylated by HIPK2, and likewise, HMGAlb, a splicing variant of HMGAla, was also phosphorylated at the corresponding sites, Thr-41 and Thr-66. The biological activities of the HMGA1 proteins are highly regulated by their post-translational modifications, including acetylation, methylation, and phosphorylation, which are dynamic and respond rapidly to extracellular stimuli. The effect of the HMGA1-phosphorylation mediated by HIPK2 was to decrease the binding affinity to DNA, which is consistent with the notion that the second AT-hook in HMGAla is crucial for DNA binding. ${ }^{59}$ Moreover, HMGAla/HIPK2 complex was also identified together STAT3 interacting protein 1 (StIP1) exerting a role in

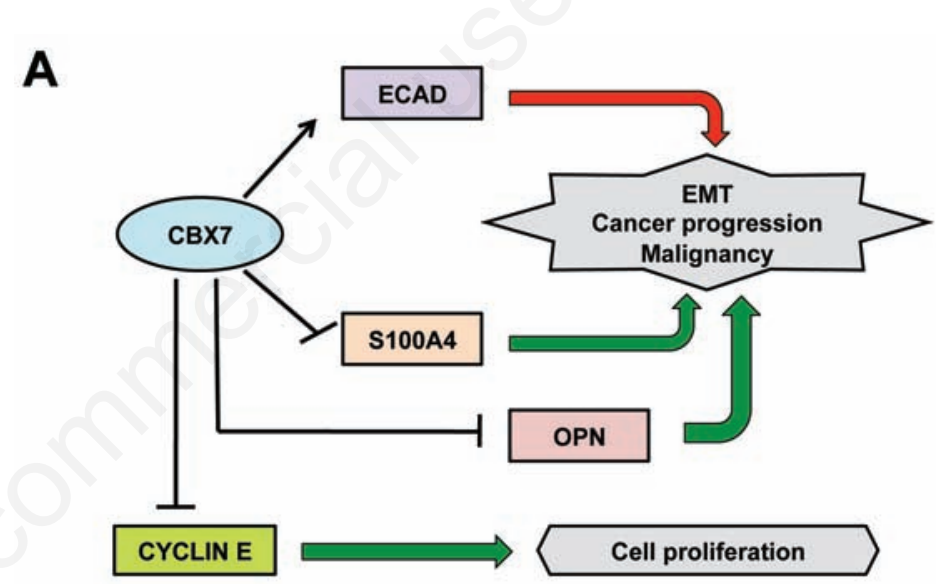

B
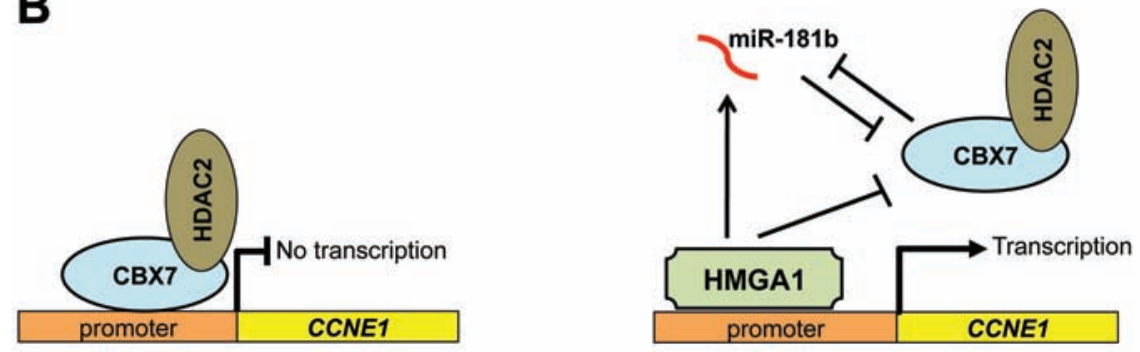

Figure 1. The central role played by the High Mobility Group A-1/chromobox protein homolog 7 (HMGA1/CBX7) pathway in cancer. A) The CBX7 inactivation during cancer progression leads to the dysregulation of several important genes, some of which are involved in the Epithelial-Mesenchymal Transition (E-cadherin, S100A4 and Osteopontin-OPN); B) HMGA1 induction in cancer is able to interfere with CBX7 function by a direct molecular interaction, which leads to the displacement of CBX7 from the cyclin E gene (CCNE1) promoter. Moreover, HMGA1 is able to repress the expression of CBX7 by directly acting on its promoter or by inducing miR-181b that, in turn, targets CBX7 for post-transcriptional repression. On the other hand, in this novel pathway, CBX7 acts repressing the expression of miR-181b, establishing a critical feedback loop. Black and green arrows: activation; red arrow and black lines: inhibition. 
HIPK2 has a crucial role other than in regulating cell growth also in regulating apoptosis during development and in response to genotoxic stress, being one of most important regulator of p53 protein. ${ }^{64,65}$ Most aspects of HIPK2regulated apoptosis rely on its ability to reprogram the transcriptional response. DNA-damaging agents can either lead to cell cycle arrest, or alternatively, when the damage exceeds the cellular repair capacity, to cell death. The death response is associated with wide changes in gene expression. The proapoptotic effects of HIPK2 are mainly mediated by its interaction with p53. Apoptotic doses of DNA-damaging agents allow HIPK2-mediated phosphorylation of human p53 at Ser-46 and of the murine homolog at Ser-58. ${ }^{66}$ This modification promotes p53 acetylation by CREB-binding protein and redirects the transcription factor p53 to proapoptotic target genes (p53AIP1, PIG3, Bax, Noxa, and KILLER/DR5). Importantly, the proapoptotic functions of HIPK2 are not limited to the p53 pathway. HIPK2 also plays a role in TGF- $\beta$ signalling, where it is important for JNK activation and JNK-mediated apoptosis. ${ }^{67}$ Recently, HIPK2 has also been reported to antagonize $L E F / \beta$ catenin signalling, by binding to Axin, and an Axin-HIPK2-p53 complex was reported to activate proapoptotic p53 target genes. ${ }^{68}$

We have concentrated our attention on the regulation of p53-dependent apoptosis since we have identified, by the screening of an antibody array, the p53 protein as an important HMGA1 interactor. ${ }^{24}$ HMGA1 binds p53 in vitro and in vivo, and interferes with the p53-mediated transcription of p53 effectors Bax and p2 $1^{\text {wafl }}$, while it cooperates with p53 to activate transcription of the p53 inhibitor mdm2, thus inhibiting its apoptotic activity. ${ }^{24}$ These observations were subsequently confirmed and extended to members of the p53 family by Frasca and coauthors. ${ }^{37}$ Furthermore, HIPK2 is able to revert the inhibitory activity of HMGA1 on the p53 effector promoters, and plays an important role also in the regulation of another anti-apoptotic gene, Bcl-2, antagonizing p53-mediated repression of Bcl-2 expression. ${ }^{69}$ In particular, HMGA1 proteins are able to abolish the repression exerted by p53-Brn-3a cooperation on $\mathrm{Bcl}-2$ promoter activity displacing HIPK2 ${ }^{69}$ Consistently, HMGA1 is able to inactivate HIPK2 by removing it from the cell nucleus and retaining it in the cytoplasm thus inhibiting p53 apoptotic function decreasing its phosphorylation at Ser-46 in wild-type p53expressing human breast carcinomas (Figure 2B).$^{70}$ This mechanism might account for the progression of neoplasms in which p53 apoptotic activity is impaired, notwithstanding the absence of p53 gene mutations and/or deletions. A similar mechanism of action was very recently reported for the potentially leuke- mogenic PEBP2 $\beta$-SMMHC fusion protein, which prevents AML1/RUNX1 activation by targeting HIPK2 to cytoplasmic filaments. ${ }^{71}$

However, human cancer cells show some tendency to inactivate HIPK2 also through other mechanisms. For instance, HIPK2 is transcriptionally downregulated in thyroid and breast carcinomas. ${ }^{72}$ In addition, HIPK2 was found to be mutated in a very small subset (2 out of 130 cases) of acute myeloid leukemia and myelodyplastic syndrome patients, ${ }^{73}$ although these rare mutations do not clearly support a causative role of HIPK2 in the pathogenesis of these tumors. More recently, Lavra and coworkers have been demonstrated allelic loss at the HIPK2 gene locus in $37.5 \%$ of papillary thyroid carcinoma PTCs. ${ }^{74}$ In this regard, it is relevant to note that the HIPK2 gene locus is located in a region where the presence of fragile sites has been reported. ${ }^{75}$

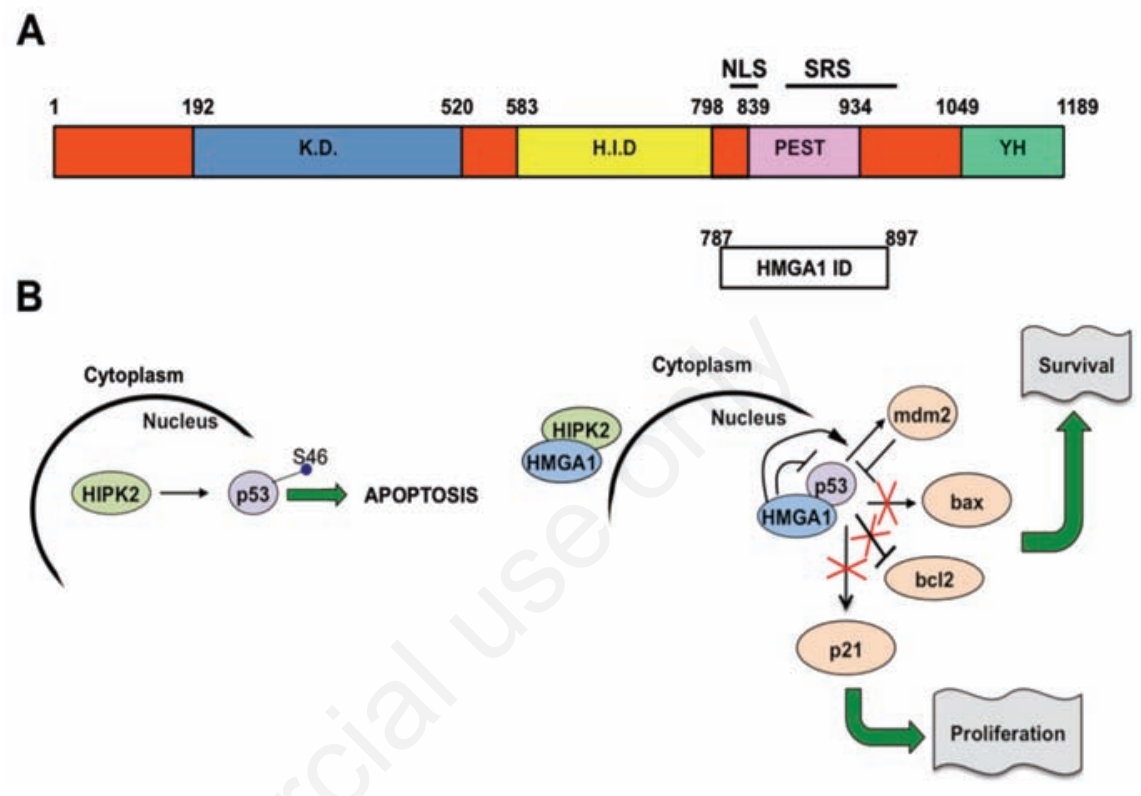

Figure 2. A) Structural diagram of the Homeodomain-interacting protein kinase-2 (HIPK2) coding sequence and the portion interacting with High Mobility Group A 1 (HMGA1) protein. The first row of numbers denotes the amino acid residues that delimit the $\mathrm{N}$-terminal region, the kinase domain (KD), the homeoprotein-interaction domain (HID), the PEST region and the $\mathrm{COOH}$-terminal region rich in tyrosine and histidine amino acids (YH domain) of mouse HIPK2. NLS: nuclear localization signal; SRS: nuclear speckle retention signal; B) Schematic representation of p53 inhibition by HMGA1 protein. In response to DNA damage, 53 induces either cell-cycle arrest or apoptosis. p53 phosphorylation at Ser- 46 by HIPK 2 is one determinant of the outcome because it occurs only after severe, nonrepairable DNA damage that irreversibly drives cells to apoptosis. HMGA1 overexpression inhibits the p53 apoptotic function by two main mechanisms: i) it promotes HIPK2 relocalization from the nucleus to the cytoplasm; ii) it directly binds to $\mathrm{p} 53$ and interferes with its mediated transcription of apoptotic or antiapoptotic (bax and bcl-2) and cell-cycle arrest (p21) effectors, while it cooperates with $p 53$ in the transcriptional activation of the $p 53$ inhibitor MDM2.

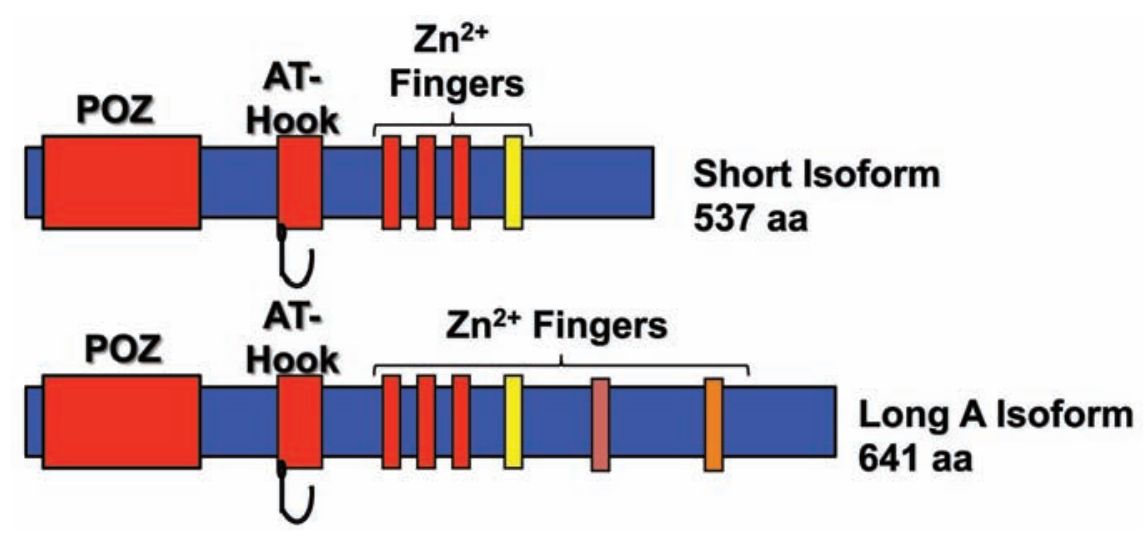

Figure 3. Schematic representation of the two most frequent isoforms of PATZ. The three characteristic domains are indicated. 
All these findings strongly suggest that HIPK2 represent a new tumor suppressor gene and may constitute a new potential promising diagnostic marker and therapeutic target.

\section{PATZ}

Another very interesting HMGA-interacting protein is PATZ, also named MAZR, ZSG or ZNF278. ${ }^{33,76,77}$ It exists in four isoforms that share a common modular structure consisting of a POZ domain, two AT-hooks and four to seven Kruppel zinc fingers (Figure 3). All these domains are characteristic of protein factors involved in gene transcription by interacting with a number of other proteins. Indeed, PATZ is a transcriptional regulatory factor that may function either as activator or repressor depending upon the cellular context: it has been reported to either activate or repress c-myc, ${ }^{33,76}$ to activate mast cell protease $6,{ }^{78}$ and to repress androgen receptor and CD8 genes. ${ }^{79,80}$ Consistent with the CD8 regulation, it has been recently shown that PATZ is an important part of the transcription factor network that controls the CD4 versus CD8 lineage fate of double-positive thymocytes. ${ }^{81}$

The PATZ1 gene, localized on human chromosome 22q12 (Figure 4), was originally cloned in the 2000 by three independent groups..$^{33,76,77}$ Mastrangelo and coworkers described a submicroscopic inversion of chromosome $22 q$ in a small round cell sarcoma with a $\mathrm{t}(1 ; 22)(\mathrm{p} 36.1 ; \mathrm{q} 12)$ translocation. The resultant chimeric transcript contained exon 8 of the Ewing sarcoma gene fused in-frame to exon 1 of the PATZ gene, creating a protein with the transactivation domain of EWS fused to the zinc finger domain of PATZ. ${ }^{77}$ Subsequently, the same group found that this paracentric inversion of chromosome $22 q 12$ interrupted the UQCRH gene, with the breakpoint in intron 3 , and created fusion genes with both EWS on der(22) and PATZ1 on der(1). PCR analysis of tumor cDNA and genomic DNA detected 5-prime-UQCRH/EWS3-prime, 5-prime-PATZ1/UQCRH-3-prime, and 5-prime-EWS/PATZ-3-prime. Only 5-primeEWS/PATZ1-3-prime produced in-frame transcripts. In contrast, 5-prime-UQCRH/EWS-3prime and 5-prime-PATZUQCRH-3-prime produced out-of-frame transcripts containing premature stop codons. ${ }^{82}$ Absence of the wild-type PATZ1 mRNA in tumor tissue supports the observation that the rearrangement of the second PATZ1 allele observed in Southern blot led to a complete loss of wild-type PATZ expression. ${ }^{77}$ Kobayashi and coworkers, as well as our group, isolated PATZ by yeast 2 -hybrid screenings with the POZ domain of Bach2 or the RING finger protein-4 (RNF4) as baits, respectively. ${ }^{33,76}$ Since RNF4 is a direct partner of
HMGA1, ${ }^{76}$ we hypothesized that PATZ could also interact with HMGA proteins. Consistently, we were able to co-immunoprecipitate endogenous PATZ, HMGA1 and HMGA2 proteins in cell extracts of HEK-293 cells, demonstrating that it is a HMGA-interacting protein (Figure 5).

Different functional and genetic evidences suggest that PATZ might be directly involved in human tumors. Besides that the PATZ1 gene has been found rearranged and deleted in a Small Round Cell Sarcoma, ${ }^{77}$ the chromosomal region where it is located is in the FRA22B fragile site, which suffers loss of heterozygosity in several solid tumours. ${ }^{83}$ In addition, its negative activity on the proto-oncogene c-myc, ${ }^{33}$ the high predisposition of Patz1-knockout mice to develop tumors (Pero et al. submitted for publication), as well as the downregulation or loss of PATZ expression in some human tumors (Fedele M, unpublished data), are consistent with a putative tumor suppressor role for the PATZ1 gene. On the other side, it has been also shown that PATZ is capable of activating c-myc in some cellular contexts, ${ }^{76}$ is overexpressed at the mRNA level in colorectal tumors ${ }^{84}$ and its down-regulation by siRNA either blocks the growth or induces apoptosis of cell lines derived from colorectal cancer or gliomas, respectively. ${ }^{84,85}$ The controversial role of PATZ in tumorigenesis could be easily explained considering that its transcriptional modulation is highly dependent on specific molecular partners of a particular cellular context. Moreover, as for other well known chromatin transcription factors, such as the HMGA proteins, it is possible they could lead to the development of neoplastic disease either if hyper- or hypoexpressed, stressing the great importance of the correct gene dosage for these factors. Interestingly, our group has demonstrated that in tumors arising in testicular germ cells, there was a significant overexpression of PATZ. However, the PATZ protein was localized in cytoplasm rather than nucleus, suggesting that this altered localization could lead to a reduction in the putative anti-oncogenic activity of PATZ, ${ }^{86}$ but the molecular mechanisms by which PATZ mis-localization leads to tumor development, have not been clarified yet. The recent finding of PATZ shuttling from nucleus to cytoplasm, upon cAMP signaling, ${ }^{87}$ may suggest a possible mechanism. Indeed, PATZ binds the RI $\alpha$ subunit of the cAMP-dependent protein kinase in the cytoplasm, and it is known that alteration of RI $\alpha$ expression, and then of the cAMP signaling, may confer cell growth advantage ${ }^{88,89}$ Therefore, the sequestration of PATZ1 in the cytoplasm through its interaction with RI $\alpha$ would enable PATZ1 to translocate into nucleus and transactivate/ repress its target genes upon activation of the cAMP pathway.

\section{Conclusions}

In this review we concentrated our attention on three HMGA-interactors, CBX7, HIPK2 and PATZ, that have been intensively studied in our laboratories, as a mean to elucidate some of the mechanisms by which HMGA proteins are involved in neoplastic transformation. It is noteworthy that all three of them have a tumor suppressor activity, which may be impaired (as so far demonstrated for CBX7 and HIPK2) by the interaction with HMGA. Indeed, CBX7 is able to compete with HMGA for the activation of the cyclin E promoter. Therefore, the downregulation of $\mathrm{CBX7}$, associated with the HMGA overexpression, events constantly observed in

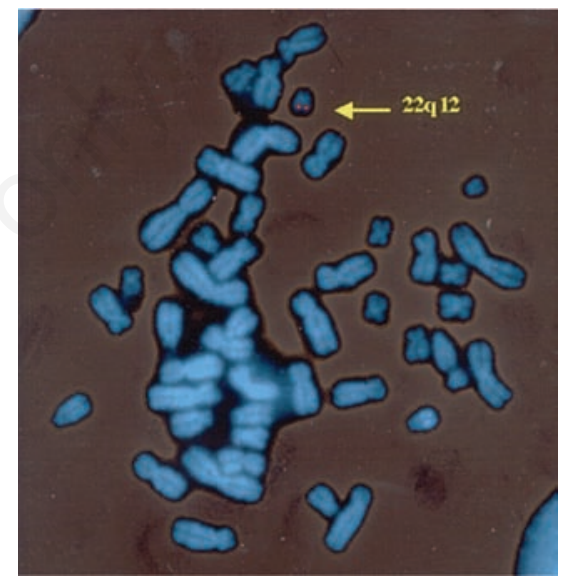

Figure 4. FISH analysis of the PATZ1 gene in human cells. The spots identify the PATZ1 alleles on chromosome 22q12.

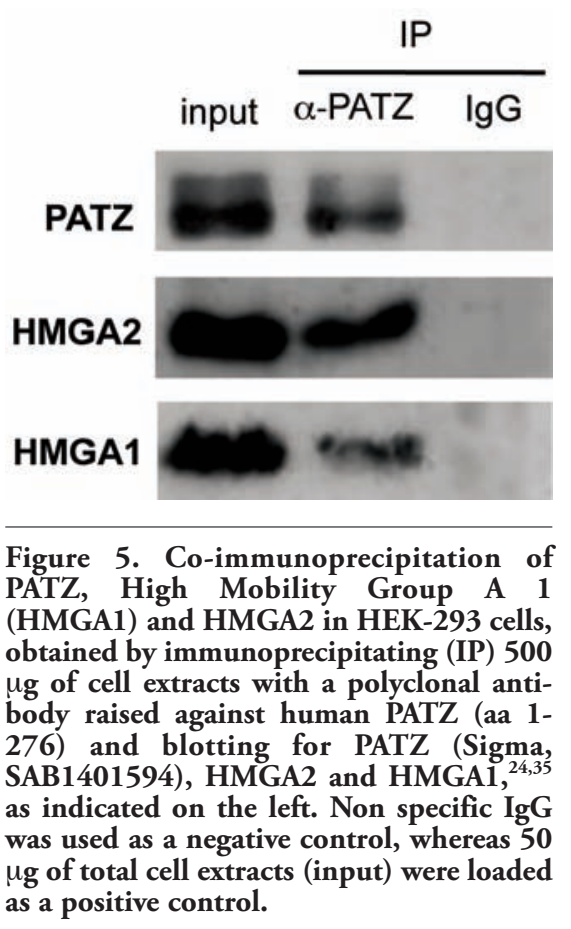


experimental and human malignancies, would allow an increased cyclin E expression that would give a cell growth advantage. In the case of HIPK2, its interaction with HMGA1 leads to delocalization of HIPK2 from nucleus to the cytoplasm, then sequestering it from the binding to p53 and thus impairing the p53-dependent transcriptional activation of pro-apoptotic genes, such as Bax. Moreover, HMGA overexpression competes with HIPK2 for the binding to $\mathrm{Bcl}-2$ promoter, thus increasing $\mathrm{Bcl}-2$ expression that would stimulate the escaping from apoptosis. As far as the role of PATZ in carcinogenesis, preliminary studies indicate that interaction with HMGA induces the loss of its tumor suppressor potential.

Therefore, it is emerging that a frequent mechanism by which HMGA proteins exert their oncogenic potential is by targeting tumor suppressors and impairing their functions, as it has been previously described for pRB and p53. ${ }^{24,35}$

However, we can also envisage the possibility that CBX7, HIPK2, PATZ and HMGA1/A2 form a multimeric complex and that HMGA1/A2 may assembly these proteins on promoters and enhancers of several genes, modulating their expression. We do not know the functional role of this complex interaction yet, but it is likely that its activity depends on the relative abundance or phosphorylation status of each component. The study of these HMGA-interactors confirms the complexity of the HMGA network that still requires further studies to be completely uncovered.

\section{References}

1. Hock R, Furusawa T, Ueda T, Bustin M. HMG chromosomal proteins in development and disease. Trends Cell Biol 2007; 17:72-9.

2. Sgarra R, Zammitti S, Lo Sardo A, et al. HMGA molecular network: From transcriptional regulation to chromatin remodeling. Bioch Bioph Acta 2010;1799:3747.

3. Chiappetta G, Avantaggiato V, Visconti R, et al. High level expression of the HMGI (Y) gene during embryonic development. Oncogene 1996;13:2439-46.

4. Rogalla P, Drechsler K, Frey G, et al. HMGI$\mathrm{C}$ expression patterns in human tissues. Implications for the genesis of frequent mesenchymal tumors. Am J Pathol 1996; 149:775-9.

5. Zhou X, Benson KF, Przybysz K, et al. Genomic structure and expression of the murine Hmgi-c gene. Nucleic Acids Res 1996;24:4071-7.

6. Rommel B, Rogalla P, Jox A, et al. HMGI-C, a member of the high mobility group family of proteins, is expressed in hematopoi- etic stem cells and in leukemic cells. Leuk Lymphoma 1997;26:603-7.

7. Nishino J, Kim I, Chada K, Morrison SJ. Hmga2 promotes neural stem cell selfrenewal in young but not old mice by reducing p16Ink4a and p19Arf Expression. Cell 2008;135:227-39.

8. Pfannkuche K, Summer H, Li 0, et al. The high mobility group protein HMGA2: a coregulator of chromatin structure and pluripotency in stem cells? Stem Cell Rev 2009;5:224-30.

9. Fusco A, Fedele M. Roles of the HMGA proteins in cancer. Nat Rev Cancer 2007;7: 899-910.

10. Hess JL. Chromosomal translocations in benign tumors: the HMGI proteins. Am J Clin Pathol 1998 109:251-61.

11. Bullerdiek J, Rommel B. Diagnostic and molecular implications of specific chromosomal translocations in mesenchymal tumors. Histol Histopathol 1999;14:116573.

12. Mayr C, Hemann MT, Bartel DP. Disrupting the pairing between let-7 and Hmga2 enhances oncogenic transformation. Science 2007;315:1576-9.

13. Hebert C, Norris K, Scheper MA, et al. High mobility group A2 is a target for miRNA-98 in head and neck squamous cell carcinoma. Mol Cancer 2007;6:5.

14. Boyerinas B, Park SM, Shomron N, et al. Identification of let-7-regulated oncofetal genes. Cancer Res 2008;68:2587-91.

15. Kaddar T, Rouault JP, Chien WW, et al. Two new miR-16 targets: caprin-1 and HMGAl, proteins implicated in cell proliferation. Biol Cell 2009;101:511-24.

16. Wei JJ, Wu X, Peng Y, et al. Regulation of HMGA1 expression by microRNA-296 affects prostate cancer growth and invasion. Clin Cancer Res 2011;17:1297-305.

17. Scala S, Portella G, Fedele M, et al. Adenovirus-mediated suppression of HMGI(Y) protein synthesis as potential therapy of human malignant neoplasias. Proc Natl Acad Sci USA 2000;97:4256-61.

18. Wood LJ, Maher JF, Bunton TE, Resar LM. The oncogenic properties of the HMG-I gene family. Cancer Res 2000;60:4256-61.

19. Fedele M, Fusco A. HMGA and cancer. Biochim Biophys Acta 2010;1799:48-54.

20. Sgarra R, Tessari MA, Di Bernardo J, et al. Discovering high mobility group A molecular partners in tumour cells. Proteomics 2005;5:1494-506.

21. Pierantoni GM, Esposito F, Giraud S, et al. Identification of new high mobility group A1 associated proteins. Proteomics 2007;7: 3735-42.

22. Sgarra R, Furlan C, Zammitti S, et al. Interaction proteomics of the HMGA chromatin architectural factors. Proteomics 2008;8:4721-32.
23. Pierantoni GM, Fedele M, Pentimalli F, et al. High mobility group I $(\mathrm{Y})$ proteins bind HIPK2, a serine-threonine kinase protein which inhibits cell growth. Oncogene 2001;20:6132-41.

24. Pierantoni GM, Rinaldo C, Esposito F, et al. High Mobility Group A1 (HMGA1) proteins interact with p53 and inhibit its apoptotic activity. Cell Death Differ 2006;13:1554-63.

25. Winter N, Nimzyk R, Bösche $\mathrm{C}$, et al. Chromatin immunoprecipitation to analyze DNA binding sites of HMGA2. PLoS One 2011;6:e18837.

26. Himes SR, Reeves R, Attema J, et al. The role of high-mobility group $\mathrm{I}(\mathrm{Y})$ proteins in expression of IL-2 and T cell proliferation. J Immunol 2000;164:3157-68.

27. Yie J, Merika M, Munshi N, et al. The role of HMG I(Y) in the assembly and function of the IFN-beta enhanceosome. EMBO J 1999;18:3074-89.

28. Pentimalli F, Palmieri D, Pacelli R, et al. HMGAl protein is a novel target of the ATM kinase. Eur J Cancer 2008;44:266879.

29. Palmieri D, Valentino T, D'Angelo D, et al. HMGA proteins promote ATM expression and enhance cancer cell resistance to genotoxic agents. Oncogene 2011;30:302435 .

30. Forzati F, Federico A, Pallante P, et al. CBX7 is a tumor suppressor in mouse and human. J Clin Invest 2012; doi: 10.1172/ JCI58620. [Epub ahead of print]

31. Bouallaga I, Massicard S, Yaniv M, Thierry F. An enhanceosome containing the Jun B/Fra-2 heterodimer and the HMG-I(Y) architectural protein controls HPV 18 transcription. EMBO Rep 2000;1:422-7.

32. Di Agostino S, Fedele M, Chieffi P, et al. Phosphorylation of high-mobility group protein $\mathrm{A} 2$ by Nek2 kinase during the first meiotic division in mouse spermatocytes. Mol Biol Cell 2004;15:1224-32.

33. Fedele M, Benvenuto G, Pero R, et al. A novel member of the BTB/POZ family, PATZ, associates with the RNF4 RING finger protein and acts as a transcriptional repressor. J Biol Chem 2000;275:7894-901.

34. Nagpal S, Ghosn C, DiSepio D, et al. Retinoid-dependent recruitment of a histone $\mathrm{H} 1$ displacement activity by retinoic acid receptor. J Biol Chem 1999;274:225638.

35. Fedele M, Visone R, De Martino I, et al. HMGA2 induces pituitary tumorigenesis by enhancing E2F1 activity. Cancer Cell 2006;9:459-71.

36. Esposito F, Pierantoni GM, Battista S, et al. Interaction between HMGAl and retinoblastoma protein is required for adipocyte differentiation. J Biol Chem 2009;284: 25993-6004.

37. Frasca F, Rustighi A, Malaguarnera R, et al. 
HMGA1 inhibits the function of p53 family members in thyroid cancer cells. Cancer Res 2006;66:2980-9.

38. Schuettengruber B, Chourrout D, Vervoort $\mathrm{M}$, et al. Genome regulation by polycomb and trithorax proteins. Cell 2007;128:73545 .

39. Scott CL, Gil J, Hernando E, et al. Role of the chromobox protein $\mathrm{CBX} 7$ in lymphomagenesis. Proc Natl Acad Sci USA 2007;104:5389-94.

40. Wu JI, Lessard J, Crabtree GR. Understanding the words of chromatin regulation. Cell 2009;136:200-6.

41. Li Q, Wang X, Lu Z, et al. Polycomb CBX7 directly controls trimethylation of histone $\mathrm{H} 3$ at lysine 9 at the p16 locus. PLoS One 2010;5:e13732.

42. Bernstein E, Duncan EM, Masui 0, et al. Mouse polycomb proteins bind differentially to methylated histone $\mathrm{H} 3$ and RNA and are enriched in facultative heterochromatin. Mol Cell Biol 2006;26:2560-9.

43. Lund AH, van Lohuizen M. Polycomb complexes and silencing mechanisms. Curr Opin Cell Biol 2004;16:239-46.

44. Pallante P, Federico A, Berlingieri MT, et al. Loss of the CBX7 gene expression correlates with a highly malignant phenotype in thyroid cancer. Cancer Res 2008;68: 6770-8.

45. Pallante P, Terracciano L, Carafa V, et al. The loss of the CBX7 gene expression represents an adverse prognostic marker for survival of colon carcinoma patients. Eur J Cancer 2010;46:2304-13.

46. Karamitopoulou E, Pallante P, Zlobec I, et al. Loss of the CBX7 protein expression correlates with a more aggressive phenotype in pancreatic cancer. Eur J Cancer 2010;46:1438-44.

47. Hinz S, Kempkensteffen C, Christoph F, et al. Expression parameters of the polycomb group proteins BMI1, SUZ12, RING1 and CBX7 in urothelial carcinoma of the bladder and their prognostic relevance. Tumour Biol 2008;29:323-9.

48. Mansueto G, Forzati F, Ferraro A, et al. Identification of a New Pathway for Tumor Progression: MicroRNA-181b UpRegulation and CBX7 Down-Regulation by HMGA1 Protein. Genes Cancer 2010;1:21024.

49. Jiang Z, Guo J, Xiao B, et al. Increased expression of miR-421 in human gastric carcinoma and its clinical association. $\mathrm{J}$ Gastroenterol 2010;45:17-23.

50. Federico A, Pallante P, Bianco M, et al. Chromobox protein homologue 7 protein, with decreased expression in human carcinomas, positively regulates E-cadherin expression by interacting with the histone deacetylase 2 protein. Cancer Res 2009;69: 7079-87.
51. Thiery JP. Epithelial-mesenchymal transitions in tumour progression. Nat Rev Cancer 2002;2:442-54.

52. Thiery JP, Sleeman JP. Complex networks orchestrate epithelial-mesenchymal transitions. Nat Rev Mol Cell Biol 2006;7:13142.

53. Boye K, Maelandsmo GM. S100A4 and metastasis: a small actor playing many roles. Am J Pathol 2010;176:528-35.

54. Shevde LA, Das S, Clark DW, Samant RS. Osteopontin: an effector and an effect of tumor metastasis. Curr Mol Med 2010;10: 71-81.

55. Bernard D, Martinez-Leal JF, Rizzo S, et al. CBX7 controls the growth of normal and tumor-derived prostate cells by repressing the Ink4a/Arf locus. Oncogene 2005;24: 5543-51.

56. Gil J, Bernard D, Martínez D, Beach D. Polycomb CBX7 has a unifying role in cellular lifespan. Nat Cell Biol 2004;6:67-72.

57. Ma Y, Fiering S, Black C, et al. Transgenic cyclin E triggers dysplasia and multiple pulmonary adenocarcinomas. Proc Natl Acad Sci U S A 2007;104:4089-94.

58. Kim YH, Choi CY, Lee SJ, Conti MA, Kim Y. Homeodomain-interacting protein kinases, a novel family of co-repressors for homeodomain transcription factors. J Biol Chem 1998;273:25875-9.

59. Giancotti V, Pani B, D'Andrea P, et al. Elevated levels of a specific class of nuclear phosphoproteins in cells transformed with v-ras and v-mos oncogenes and by cotransfection with c-myc and polyoma middle T genes. EMBO J 1987;6:19817.

60. Zhang Q, Wang Y. Homeodomain-interacting protein kinase-2 (HIPK2) phosphorylates HMGAla at Ser-35, Thr-52, and Thr77 and modulates its DNA binding affinity. J Proteome Res 2007;6:4711-9.

61. Okuda H, Manabe T, Yanagita T, et al. Novel interaction between HMGAla and StIP1 in murine terminally differentiated retina. Mol Cell Neurosci 2006;33:81-7.

62. Trapasso F, Aqeilan RI, Iuliano R, et al. Targeted disruption of the murine homeodomain-interacting protein kinase-2 causes growth deficiency in vivo and cell cycle arrest in vitro. DNA Cell Biol 2009;28:161-7.

63. Iacovelli S, Ciuffini L, Lazzari $\mathrm{C}$, et al. HIPK2 is involved in cell proliferation and its suppression promotes growth arrest independently of DNA damage. Cell Prolif 2009;42:373-84.

64. D'Orazi G, Cecchinelli B, Bruno T, et al. Homeodomain-interacting protein kinase2 phosphorylates p53 at Ser 46 and mediates apoptosis. Nat Cell Biol 2002;4:11-9.

65. Hofmann TG, Moller A, Sirma H, et al. Regulation of $\mathrm{p} 53$ activity by its interaction with homeodomain-interacting protein kinase-2. Nat Cell Biol 2002; 4:1-10.

66. Cecchinelli B, Porrello A, Lazzari C, et al. Ser58 of mouse p53 is the homologue of human Ser46 and is phosphorylated by HIPK2 in apoptosis. Cell Death Differ 2006;13:1994-7.

67. Hofmann TG, Stollberg N, Schmitz ML, Will H. HIPK2 regulates transforming growth factor-beta-induced c-Jun NH(2)-terminal kinase activation and apoptosis in human hepatoma cells. Cancer Res 2003;63:82717.

68. Rui Y, Xu Z, Lin S, et al. Axin stimulates p53 functions by activation of HIPK2 kinase through multimeric complex formation. EMBO J 2004;23:4583-94.

69. Esposito F, Tornincasa M, Chieffi P, et al. High-mobility group A1 proteins regulate p53-mediated transcription of Bcl-2 gene. Cancer Res 2010;70:5379-88.

70. Pierantoni GM, Rinaldo C, Mottolese M, et al. High-mobility group A1 inhibits p53 by cytoplasmic relocalization of its proapoptotic activator HIPK2. J Clin Invest 2007; 117:693-702.

71. Wee HJ, Voon DC, Bae SC, Ito Y. PEBP2beta/CBF-beta-dependent phosphorylation of RUNX1 and p300 by HIPK2: implications for leukemogenesis. Blood 2008;112:377787.

72. Pierantoni GM, Bulfone A, Pentimalli F, et al. The homeodomain-interacting protein kinase 2 gene is expressed late in embryogenesis and preferentially in retina, muscle, and neural tissues. Biochem Biophys Res Commun 2002;290:942-7.

73. Li XL, Arai Y, Harada H, et al. Mutations of the HIPK2 gene in acute myeloid leukemia and myelodysplastic syndrome impair AML1- and p53-mediated transcription. Oncogene 2007;26:7231-9.

74. Lavra L, Rinaldo C, Ulivieri A, et al. The loss of the p53 activator HIPK2 is responsible for galectin-3 overexpression in well differentiated thyroid carcinomas. PLoS One 2011;6:e20665.

75. Huang H, Reed CP, Mordi A, et al. Frequent deletions within FRA7G at 7q312 in invasive epithelial ovarian cancer. Genes Chromosomes Cancer 1999;24:48-55.

76. Kobayashi A, Yamagiwa H, Hoshino $\mathrm{H}$, et al. A combinatorial code for gene expression generated by transcription factor Bach2 and MAZR (MAZ-related factor) through the BTB/POZ domain. Mol Cell Biol 2000;20:1733-46.

77. Mastrangelo T, Modena P, Tornielli S, et al. A novel zinc finger gene is fused to EWS in small round cell tumor. Oncogene 2000;19: 3799-804.

78. Morii E, Oboki $\mathrm{K}$, Kataoka TR, et al. Interaction and cooperation of mi transcription factor (MITF) and myc-associat- 
ed zinc-finger protein-related factor (MAZR) for transcription of mouse mast cell protease 6 gene. $\mathrm{J}$ Biol Chem 2002;277:8566-71.

79. Pero R, Lembo F, Palmieri EA, et al. PATZ attenuates the RNF4-mediated enhancement of androgen receptor-dependent transcription. J Biol Chem 2002;277:32805 .

80. Bilic I, Koesters C, Unger B, et al. Negative regulation of $\mathrm{CD} 8$ expression via $\mathrm{Cd} 8$ enhancer-mediated recruitment of the zinc finger protein MAZR. Nat Immunol 2006;7:392-400.

81. Sakaguchi S, Hombauer M, Bilic I, et al. The zinc-finger protein MAZR is part of the transcription factor network that controls the CD4 versus CD8 lineage fate of double-positive thymocytes. Nat Immunol 2010;11:442-8.

82. Modena P, Testi MA, Facchinetti F, et al.
UQCRH gene encoding mitochondrial Hinge protein is interrupted by a translocation in a soft-tissue sarcoma and epigenetically inactivated in some cancer cell lines. Oncogene 2003;22:4586-93.

83. Burrow AA, Williams LE, Pierce LC, Wang YH. Over half of breakpoints in gene pairs involved in cancer-specific recurrent translocations are mapped to human chromosomal fragile sites. BMC Genomics 2009;10:59.

84. Tian X, Sun D, Zhang Y, Zhao S, Xiong H, Fang J. Zinc finger protein 278, a potential oncogene in human colorectal cancer. Acta Biochim Biophys Sin (Shanghai) 2008;40: 289-96.

85. Tritz R, Mueller BM, Hickey MJ, et al. siRNA Down-regulation of the PATZ1 Gene in Human Glioma Cells Increases Their Sensitivity to Apoptotic Stimuli. Cancer Ther 2008;6:865-76.
86. Fedele M, Franco R, Salvatore G, et al. PATZ1 gene has a critical role in the spermatogenesis and testicular tumours. J Pathol 2008;215:39-47.

87. Yang WL, Ravatn R, Kudoh K, Alabanza L, Chin KV. Interaction of the regulatory subunit of the cAMP-dependent protein kinase with PATZ1 (ZNF278). Biochem Biophys Res Commun 2010;391:1318-23.

88. Tortora G, Pepe S, Bianco C, et al. The RI alpha subunit of protein kinase A controls serum dependency and entry into cell cycle of human mammary epithelial cells. Oncogene 1994;9:3233-40.

89. Tortora G, Pepe S, Bianco C, et al. Differential effects of protein kinase A sub-units on Chinese-hamster-ovary cell cycle and proliferation. Int J Cancer 1994; 59:712-6. 